\title{
Structural aspects of the phonon spectra of $\mathrm{La}_{1-x} \mathrm{Sr}_{x} \mathrm{MnO}_{3}$
}

\author{
F. Mayr, Ch. Hartinger, and A. Loidl \\ Experimentalphysik V, Elektronische Korrelationen und Magnetismus, Institut für Physik, Universität Augsburg, \\ D-86135 Augsburg, Germany
}

(Received 16 March 2005; published 14 July 2005)

\begin{abstract}
The lightly doped $\mathrm{La}_{1-x} \mathrm{Sr}_{x} \mathrm{MnO}_{3}$ system was investigated using x-ray diffraction and far-infrared spectroscopy in the insulating range for $x \leqslant 0.125$ on single crystalline samples. Special emphasis was set on the evolution of the Jahn-Teller (JT) distortion. With increasing doping concentration $x$ the optical phonon excitations broaden and merge into three groups, consistent with a weakening of the orthorhombic JT distortion. The temperature and doping dependence of the phonon anomalies are related to the complex phase diagram, which is governed by electronic, magnetic, and structural degrees of freedom. Indications for a magnonphonon coupling are found for specific phonon modes.
\end{abstract}

DOI: 10.1103/PhysRevB.72.024425

PACS number(s): 63.20.-e, 78.20.-e, 64.70.Kb

\section{INTRODUCTION}

The physics of manganites plays a prominent role in recent state research. It arises from the rich complexity of the involved interactions, in particular between structural, electronic, magnetic, and orbital degrees of freedom. For specific doping concentrations and temperature regimes, the competing internal forces are approximately balanced, which leads to a vast variety of phases as a function of doping and/or temperature. The most popular example is the colossal magnetoresistance effect, where spin and charge correlations are mediated through the lattice. ${ }^{1,2}$

The structure of the low-doping regime of the series $\mathrm{La}_{1-x} \mathrm{Sr}_{x} \mathrm{MnO}_{3}(0 \leqslant x \leqslant 0.15)$ is characterized by a successive change between different modifications of the orthorhombic perovskite cell with Pnma symmetry ( $O$ phase). The JahnTeller (JT) distortion dominates the low-temperature ground state $\left(O^{\prime}\right.$ phase) and is responsible for the complex structural phase diagram. ${ }^{3-5}$ Antiferroorbital ordering within the $a b$ plane occurs in the JT distorted $O^{\prime}$ phase. ${ }^{6}$ In a narrow range $0.1 \leqslant x \leqslant 0.16$ a ferromagnetic insulating phase, probably with a different orbital order compared to the $O^{\prime}$ phase, ${ }^{7,8}$ is formed at low temperatures $\left(O^{\prime \prime}\right.$ phase), where charge ${ }^{9,10}$ and orbital $^{11}$ ordering are supposed to coexist.

Optical spectroscopy serves as an excellent tool for monitoring the variety of emerging physical effects due to its sensitivity to different excitation processes. While in the high-energy region above $1 \mathrm{eV}$, the spectra are governed by electronic transitions; ${ }^{12-14}$ the low-energy part is directly sensitive to structural changes via the phonon dynamics. As the lattice is a key point in understanding the principal manganite phenomena, a close inspection of the phonons is inevitable. For example, in the metallic phase for $x=0.33$ an inhomogeneous dynamical JT distorted state ${ }^{15}$ and a finite electron-lattice coupling ${ }^{16}$ were observed via phonon spectroscopy.

A number of previous publications about the phonon properties and the structure of low-doped perovskite manganites exist. The infrared (IR) active lattice modes have been analyzed theoretically ${ }^{17,18}$ and experimentally, ${ }^{19-24}$ with most effort spent on undoped $\mathrm{LaMnO}_{3}$. Takazawa et al. analyze the doping and temperature dependence of the phonon spectra in the narrow range $x=0.1-0.125$ using Lorentzian oscillator fits to their reflectivity data. While the sample with $x$ $=0.1$ shows no specific phonon anomalies, for $x=0.125$ they find indications of a strong correlation between the bendingtype lattice vibration and the orbital ordering at low temperatures.

Temperature-dependent Raman spectra ${ }^{25-27}$ revealed irregularities in the shift of some Raman active modes close to the antiferromagnetic ordering temperature and gave hints about an existing coupling between lattice and magnetism. For $\mathrm{La}_{0.7} \mathrm{Sr}_{0.3} \mathrm{MnO}_{3}$ the frequency of an $A_{1 g}$-mode was found to exhibit a rather unusual doping dependence opposite to that expected from the difference in the atomic weight of $\mathrm{Sr}$ and La. Neutron scattering experiments ${ }^{8,28}$ probed the dispersion relation of the phonons, where a splitting of the spin wave spectrum at $T_{O^{\prime} O^{\prime \prime}}$ is observed, proving the magnetoelastic character of this phase boundary.

The lattice symmetry and the temperature evolution of the lattice dimensions have also been monitored by various authors. ${ }^{11,29-34}$ The structural phase diagram for $0.08 \leqslant x$ $\leqslant 0.125$ was investigated in detail by high-resolution synchrotron x-ray diffraction (XRD) by Cox et al. ${ }^{35}$ For low temperatures they find two lower-symmetry structural regions having monoclinic and triclinic symmetry.

Recently, the temperature dependence of the lattice constants was found to show anomalies connected to the JT distortion. ${ }^{36}$ As the phonons are closely related with the structural symmetry, similar effects are expected for the IR spectra of low-doped manganites. Phonon spectroscopy can reveal distinct information about the different phases and the structural changes at the phase boundaries. Nevertheless, up to now no systematic study exists concerning the temperature and doping dependence of single crystalline samples in this doping range, dealing with the connection of phonons with the structure.

In this paper we report on optical spectroscopy measurements on $\mathrm{La}_{1-x} \mathrm{Sr}_{x} \mathrm{MnO}_{3}(0 \leqslant x \leqslant 0.125)$, where all samples are insulating. We pay special attention to the phonon region of the spectra and investigate their variation with temperature and doping. Although the structural properties of low- 
doped manganites were analyzed with XRD in several prior publications, we nevertheless provide XRD data of our single crystals. First, the observed structures and transition temperatures differ to some extent in literature, which makes it inevitable to determine the structural properties of the same samples, which are used for the optical measurements. Second, optical phonon spectroscopy probes the microscopic structures of the unit cell as particular motions of the atoms are investigated for the distinct vibrational modes. To correlate the observed anomalies in the temperature dependence of the phonons to structural properties of the unit cell, especially the Jahn-Teller distortion, the unit cell properties have to be known. The connection between the complex structural phase diagram and the phonon properties is discussed in detail.

\section{EXPERIMENTAL DETAILS}

Single crystals of $\mathrm{La}_{1-x} \mathrm{Sr}_{x} \mathrm{MnO}_{3}(x=0,0.025,0.05,0.075$, 0.1 , and 0.125 ) were grown by the floating zone method with radiation heating as described elsewhere. ${ }^{37}$ Laue pictures revealed a twinned structure of the crystals. To prepare the samples for the optical measurements, they were cut into $\approx 2 \mathrm{~mm}$ thick slices and carefully polished to obtain optical flat surfaces.

The crystal structure and lattice constants were determined by powder $\mathrm{x}$-ray diffraction, using monochromatic $\mathrm{CuK} \alpha_{1}$-radiation $(\lambda=1.540598 \AA)$ on a STOE diffractometer with $\omega-2 \theta$ geometry. The temperature dependence of the unit-cell dimensions were monitored in the range from 90 to $400 \mathrm{~K}$ through placing the samples in a nitrogen gas stream. The magnetic dc and ac susceptibilities were measured with a commercial 5T-SQUID magnetometer from Quantum Design. The dc resistivity was obtained with a standard four-point method.

In the infrared (IR) regime the temperature-dependent reflectivity has been measured using a Bruker IFS 113v Fourier transform spectrometer in the spectral range from 40 to $700 \mathrm{~cm}^{-1}$. For frequencies from 500 to $40000 \mathrm{~cm}^{-1}$ a Bruker $66 \mathrm{v} / \mathrm{S}$ FT-spectrometer was used. All spectra were recorded with a resolution of $1 \mathrm{~cm}^{-1}$. The samples were placed in the exchange gas of a ${ }^{4} \mathrm{He}$-bath cryostat, which was equipped with polypropylene windows for the far-IR, KRS-5 windows for the mid- and near-IR and quartz windows for the VIS/ UV-range. The optical conductivity spectra were obtained by Kramers-Kronig analysis of the reflectivity data. For this analysis, the low-energy part was extended with a constant approximation to zero frequency and for the high-energy range a smooth $\nu^{-1.5}$ extrapolation to zero reflectivity was used.

\section{RESULTS AND DISCUSSION}

In the low-doping range $x<0.2$ the phase diagram of $\mathrm{La}_{1-x} \mathrm{Sr}_{x} \mathrm{MnO}_{3}$ reveals four structural (one rhombohedral and three orthorhombic), four magnetic [paramagnetic (PM), antiferromagnetic (AF), canted antiferromagnetic $(\mathrm{CAF})$ and ferromagnetic (FM)] and two electronic (insulating and conducting) phases.
TABLE I. Phase-transition temperatures (in Kelvin) of the different $\mathrm{La}_{1-x} \mathrm{Sr}_{x} \mathrm{MnO}_{3}$ single crystals extracted from x-ray diffraction, dc resistivity, and magnetic susceptibility measurements. $T_{\mathrm{CA}}$ denotes the CAF transition; $T_{R O}, T_{O O^{\prime}}$, and $T_{O^{\prime} O^{\prime \prime}}$ describe the structural transitions between the $R, O, O^{\prime}$, and $O^{\prime \prime}$ phases. The values for $T_{R O}$ for $x=0,0.025$, and 0.05 have been taken from Refs. 34 and 38.

\begin{tabular}{lrrrr}
\hline \hline $\mathrm{La}_{1-x} \mathrm{Sr}_{x} \mathrm{MnO}_{3}$ & $T_{\mathrm{CA}}$ & $T_{R O}$ & $T_{O O^{\prime}}$ & $T_{O^{\prime} O^{\prime \prime}}$ \\
\hline$x=0$ & 136 & 1010 & 780 & \\
$x=0.025$ & 132 & 890 & 655 & \\
$x=0.05$ & 130 & 750 & 550 & \\
$x=0.075$ & 125 & 640 & 400 & \\
$x=0.1$ & 148 & 550 & 340 & 104 \\
$x=0.125$ & 180 & 460 & 265 & 140 \\
\hline \hline
\end{tabular}

While pure $\mathrm{LaMnO}_{3}$ has an AF spin order below $140 \mathrm{~K}$, the magnetic ground state for $x<0.1$ supports a CAF structure. In the range $x \gtrsim 0.1$, a FM low-temperature state is established, which is accompanied by an orbital order in the range $0.1 \leqq x \leqq 0.16\left(O^{\prime \prime}\right.$ phase $)$. $^{3,4,11}$ The relative spin orientation of neighboring $\mathrm{Mn}$ ions affects the structural stabilization energy, ${ }^{29}$ and a strong coupling between the spin and structural degrees of freedom exists.

The various transition temperatures for the investigated samples were extracted from x-ray diffraction, dc resistivity, and magnetic susceptibility measurements, which are summarized in Table I. As an example Fig. 1 shows the x-ray diffraction pattern for $x=0.1$ at $300 \mathrm{~K}$ (lower part) with focus

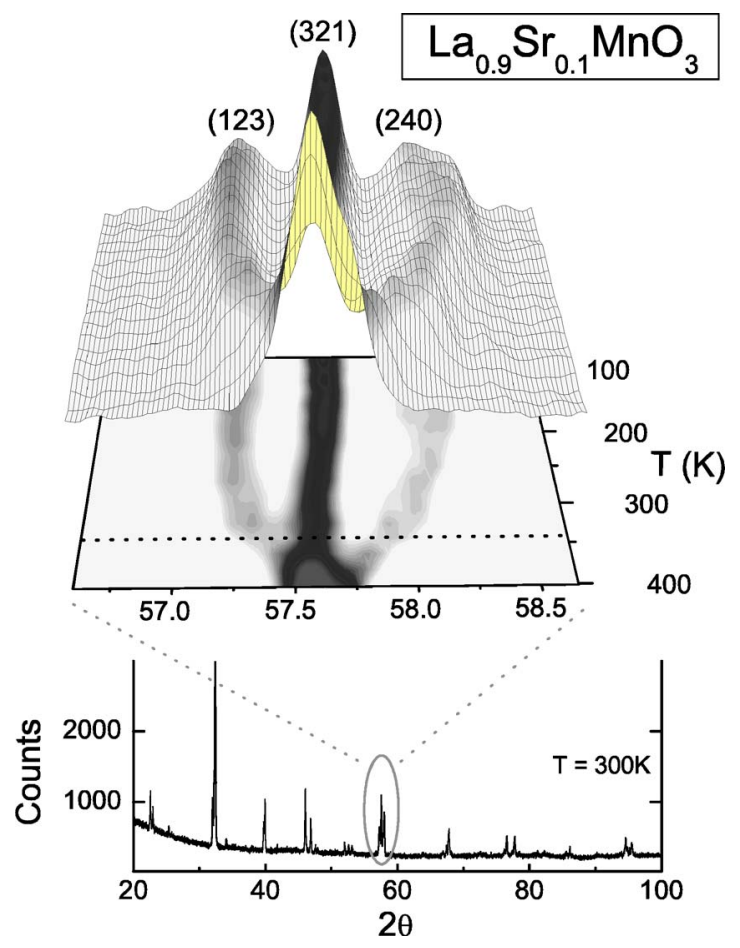

FIG. 1. (Color online) X-ray diffraction pattern of $\mathrm{La}_{0.9} \mathrm{Sr}_{0.1} \mathrm{MnO}_{3}$ at $300 \mathrm{~K}$ (lower part). The three-dimensional graph (upper part) shows the temperature evolution of the (123), (321), and (240) reflection peaks. 


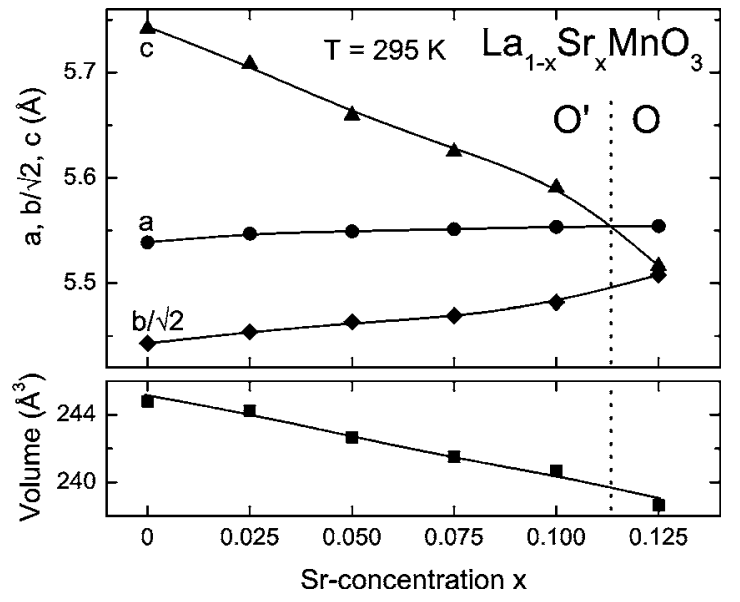

FIG. 2. Room-temperature lattice parameters (upper frame) and unit-cell volume (lower frame) of the series $\mathrm{La}_{1-x} \mathrm{Sr}_{x} \mathrm{MnO}_{3}$. The $O O^{\prime}$ transition is located at about $x \approx 0.11$ (vertical dotted line).

on the temperature evolution of the $(123) /(321) /(240)$ reflections (upper part). The spectrum can be indexed with reflections from orthorhombic Pnma planes, revealing a purely single-phase structure. Below about $340 \mathrm{~K}$ the $(123) /(321) /(240)$ Bragg-reflections around $57.5^{\circ}$ show an increasing splitting into three distinct peaks, indicating a reduction of symmetry of the unit cell. This is a consequence of the JT distortion of the $\mathrm{MnO}_{6}$ octahedra, which manifests itself also in a change of the unit cell parameters (cf. Fig. 5). In Ref. 35 high-resolution synchrotron x-ray data for $\mathrm{La}: \mathrm{SrMnO}_{3}$ are documented for $\mathrm{Sr}$ concentrations $0.08 \leqslant x$ $\leqslant 0.125$. While at room temperature the overall agreement is very good, Ref. 35 reveals lower symmetry phases for concentrations close to $x=0.11$. Specifically, a sharp transition from a monoclinic to a triclinic phase has been identified coinciding with the occurrence of the ferromagnetic insulating phase. In our work these low-symmetry phases could not be identified, neither by $\mathrm{x}$-ray diffraction nor by reflectivity measurements of the phonon modes in the FIR range. We conjecture that if these low-symmetry phases would occur in our samples, the distortions are so small that the phonons still reveal an overall orthorhombic symmetry behavior.

In general, at elevated temperatures the system is stable in the rhombohedral $R \overline{3} c$ structure ( $R$ phase). Upon lowering the temperature, the structure changes to orthorhombic Pnma symmetry ( $O$ phase). With further decreasing temperature, an additional long-range Jahn-Teller distortion occurs, where the $\mathrm{Mn}-\mathrm{O}$ bond lengths of the $\mathrm{MnO}_{6}$ octahedra are modified. ${ }^{36}$

\section{A. Structural aspects}

To investigate the structural effect of Sr doping, we determined the unit cell dimensions from the x-ray diffraction patterns of all single crystals used in this investigation. The evolution of the room-temperature lattice parameters with $\mathrm{Sr}$ concentration $x$ is depicted in Fig. 2. In the upper frame the lattice constants $a$ (circles), $b / \sqrt{2}$ (diamonds), and $c$ (triangles) are shown, while in the lower frame the unit cell volume (squares) is plotted.
As $a$ and $b / \sqrt{2}$ increase slightly with increasing $x$, the $c$ axis shows quite a substantial decrease, which is a consequence of the reduced distortion of the $\mathrm{MnO}_{6}$ octahedra. The significant feature is the crossing of $a$ and $c$ at about $x$ $\approx 0.11$, which indicates the disappearance of the JT distortion and the symmetry change from $O^{\prime}$ to $O$. In the nondistorted $O$ phase, $a, b / \sqrt{2}$, and $c$ differ only slightly. The unit cell volume decreases almost linearly up to $x=0.125$ and reveals no distinct anomaly at the structural phase transition at $x \approx 0.11$. These findings are in excellent agreement with high-resolution synchrotron XRD results for $0.08 \leqslant x$ $\leqslant 0.125 .^{35}$

The far-IR range of the optical conductivity $\sigma^{\prime}$ is dominated by the phonon excitations, which are in particular sensitive to structural changes. A group theoretical analysis of the $O$ phase was performed by Smirnova. ${ }^{18}$ For a lattice with Pnma symmetry a total of 25 infrared active phonon modes should be visible. We find 17 different modes at $295 \mathrm{~K}$ and 20 phonon lines at $4 \mathrm{~K}$. Even though not all modes are resolved, which might be due to a small oscillator strength or overlapping with neighboring modes, the optical spectra clearly confirm the orthorhombic Pnma structure of $\mathrm{LaMnO}_{3}$.

Although the actual lattice symmetry of the investigated samples deviates from the simple cubic perovskite structure, the optical phonons can be interpreted in terms which originate from this simple symmetry. This approach is justified by the fact that the unit cell of the cubic Pm3m perovskite structure is the main component of the orthorhombic unit cell. The Pnma unit cell can be obtained from the cubic one by a combined rotation of the $\mathrm{MnO}_{6}$ octahedra along the (100) and (110) direction, which results in a buckling of the octahedra along the $b$ axis. ${ }^{39}$ Increased doping reduces this buckling, and the unit cell gains a more cubic character.

For the cubic Pm3m symmetry, only three phonon bands are IR active, which can be related to specific types of lattice vibrations. ${ }^{40}$ The low-energy external mode is characterized by a relative motion of the $\mathrm{MnO}_{6}$ octahedra against the surrounding $\mathrm{La} / \mathrm{Sr}$ cage. The bending mode vibration is sensitive to a change in the $\mathrm{Mn}-\mathrm{O}$ bond angles, while the high energy stretching mode is affected by the $\mathrm{Mn}-\mathrm{O}$ bonding lengths. A close inspection of the results of the theoretical analysis of the orthorhombic modes ${ }^{18}$ reveals that the dominant character of the different modes can be grouped in external (low-frequency), bending (mid-frequency), and stretching (high-frequency) type of vibrations. This justifies our description in terms of the cubic modes in the following.

A symmetry change from orthorhombic to cubic reduces the number of IR-active phonon modes. The crossover from orthorhombic to cubic-like behavior with decreasing orthorhombic distortion can be seen in Fig. 3. Here we show in a three-dimensional plot the optical conductivity in the phonon regime for the various single crystals at room temperature. At first sight, the number of phonons decreases with increasing doping and the spectra reveal a rough classification of the phonons into the three cubic groups. Around $170 \mathrm{~cm}^{-1}$ the external mode is present. In the mid-range, around $350 \mathrm{~cm}^{-1}$, the broad group of bending type is visible. The stretching mode appears in the high energy end at about $570 \mathrm{~cm}^{-1}$.

This figure impressively documents the weakening of the JT distortion, which is strongest for $x=0$. As a general trend, 


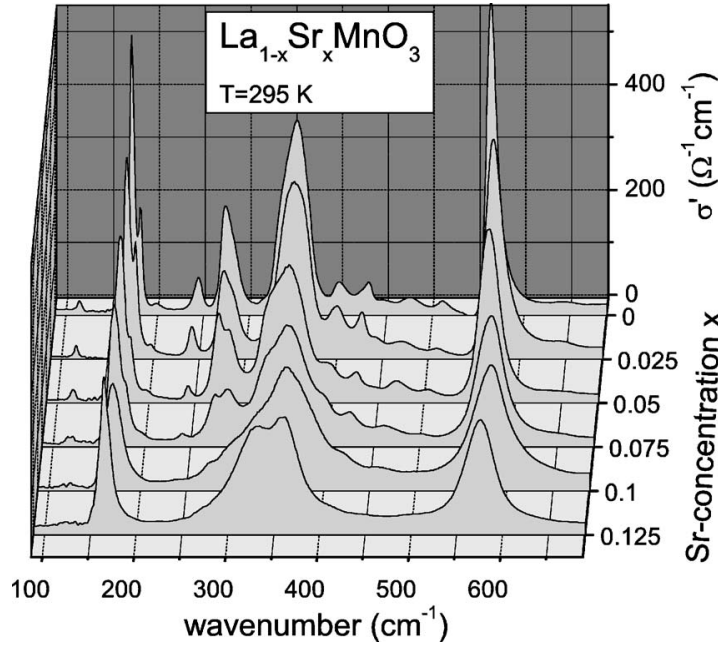

FIG. 3. Optical conductivity $\sigma^{\prime}$ of $\mathrm{La}_{1-x} \mathrm{Sr}_{x} \mathrm{MnO}_{3}$ at $295 \mathrm{~K}$ for various $\mathrm{Sr}$ concentrations $x$ (right axis).

the spectra show a reduced fine structure with increasing $\mathrm{Sr}$ content. The spectrum for the pure compound displays 17 distinct phonon modes, while the $x=0.125$ doped sample reveals only 4 separate vibrational excitations. This is a clear experimental evidence that the orthorhombic distortion, which is responsible for the small side bands, weakens upon Sr doping. In addition, the doping-induced disorder due to the changed mass, charge, and lattice parameters tends to broaden the phonon bands. This might also be a reason for the decreasing number of bands, because small neighboring bands may overlap due to this broadening effect.

To investigate the consequences of the modified JT distortion and the changes in lattice parameter and ionic mass, Fig. 4 shows the $\mathrm{Sr}$ concentration $x$ dependence of the phononmode eigenfrequencies of the three main bands. Plotted are the values at 295 and $200 \mathrm{~K}$, respectively. For the former temperature, an $O^{\prime} O$ transition at $x \approx 0.11$ occurs, while for the latter all samples are in the JT-distorted $O^{\prime}$ phase.

The external mode should be less influenced by the inner geometry of the $\mathrm{MnO}_{6}$ octahedra. The charge replacement of $\mathrm{La}^{3+}$ via doping with $\mathrm{Sr}^{2+}$ weakens the force constant for this mode and is accountable for the decrease of $\nu_{1}$ with $x$ (lower panel of Fig. 4). On the contrary, the bending and the stretching mode vibrations are more sensitive to the $\mathrm{MnO}_{6}$ geometry. The bending mode (middle panel of Fig. 4) shows at $200 \mathrm{~K}$ a slight linear increase with $x$. At room temperature (RT), a sharp rise around $x \approx 0.11$ is established, corresponding to the changes in the $\mathrm{MnO}$ bonding angles.

A similar behavior can be expected for the stretching mode. At room temperature, a maximum is found in the eigenfrequency $\nu_{3}$ vs $x$ curve at the $O^{\prime} O$ transition. The decrease of $\nu_{3}$ for $x=0.125$ seems to be related to the structural difference between the $O^{\prime}$ and $O$ phases. Nevertheless, without this transition at $200 \mathrm{~K}$, a similar maximum shows up at $x=0.1$. Probably the simple interpretation in terms of the cubic phonon vibrations is inappropriate for this mode and one has to take into account the more complex orthorhombic phonon vibrations. For the two possible orthorhombic candidates in Fig. 5 of Ref. $18\left(B_{1 u}\right.$ at $579 \mathrm{~cm}^{-1}$ and $B_{2 u}$ at $562 \mathrm{~cm}^{-1}$ ) the atoms vibrate almost entirely within the $a-c$

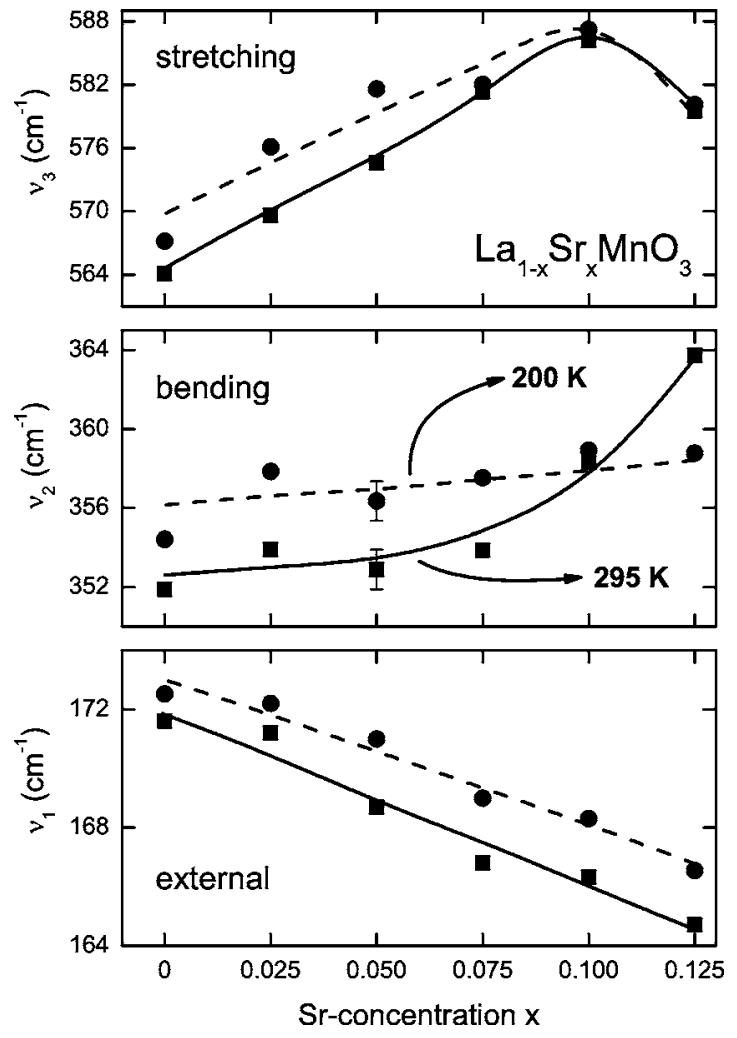

FIG. 4. Eigenfrequencies of the three main phonon bands of $\mathrm{La}_{1-x} \mathrm{Sr}_{x} \mathrm{MnO}_{3}$ at $295 \mathrm{~K}$ (square symbols) and at $200 \mathrm{~K}$ (circle symbols) as a function of the $\mathrm{Sr}$ concentration $x$. The lines serve as a guide to the eyes.

plane. Therefore it seems very likely that $O$ and $O^{\prime}$ are structurally similar within this plane, and the cardinal effect of the JT distortion is along the $b$ axis.

\section{B. Magnetoelastic coupling}

The temperature dependence of the lattice parameters of $\mathrm{La}_{0.9} \mathrm{Sr}_{0.1} \mathrm{MnO}_{3}$ is plotted in the upper panel of Fig. 5. At 400 $\mathrm{K}$, in the $O$ phase, $a, b / \sqrt{2}$, and $c$ differ by $\approx 0.9 \%$, indicating only slight variations in the $\mathrm{Mn}-\mathrm{O}$ distance. In the JTdistorted $O^{\prime}$ phase the three axes show an increased splitting, which reaches its maximum value of $\approx 2.6 \%$ at $100 \mathrm{~K}$. This behavior is a consequence of the different bond lengths in the $\mathrm{MnO}_{6}$ octahedra. ${ }^{30,31}$ Similar results were found for $x=1 / 8.41,42$ The authors observe a reduction in the splitting of the $\mathrm{MnO}$ bond lengths in the less distorted $O^{\prime \prime}$ phase. As we find no clear indications of a relaxation of the lattice parameters down to about $90 \mathrm{~K}$, it seems that in $\mathrm{La}_{0.9} \mathrm{Sr}_{0.1} \mathrm{MnO}_{3}$ this phase is reached at lower temperatures. It is worth mentioning that the ferromagnetic order, which accompanies the transition into the $O^{\prime \prime}$ phase, ${ }^{29,30}$ already sets in above $100 \mathrm{~K}$. In the left inset of the lower panel of Fig. 5 a clear peak in the magnetic ac susceptibility is found at $T_{O^{\prime} O^{\prime \prime}}=104 \mathrm{~K}$.

Although the transition $O / O^{\prime}$ is of structural origin, it is clearly connected to the magnetic and electric transport properties of the sample. In the lower panel of Fig. 5 we show the inverse magnetic susceptibility, $1 / \chi$ (right axis), and the 


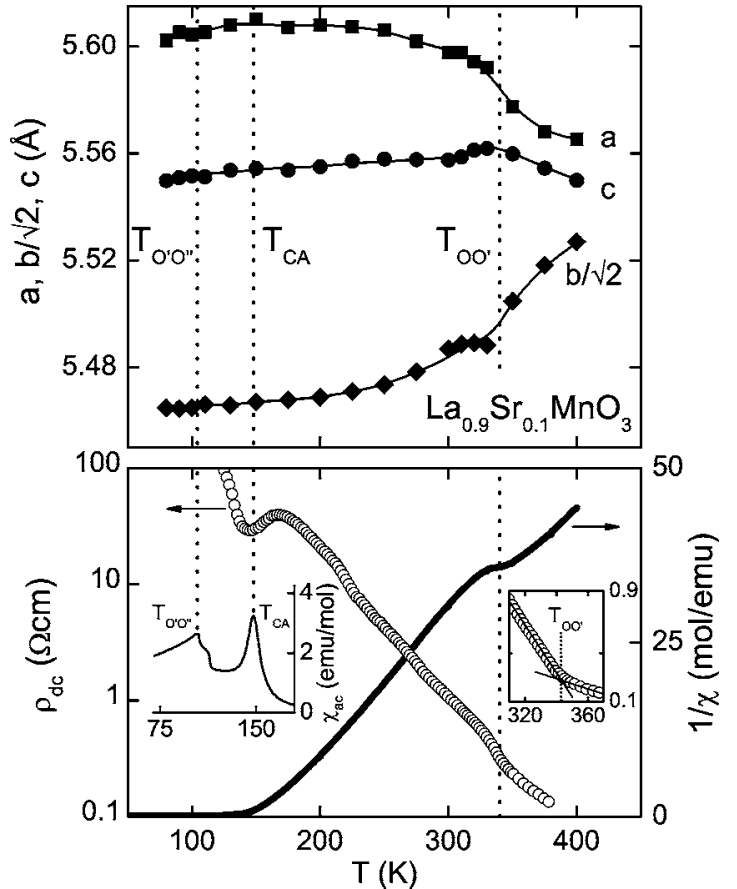

FIG. 5. Upper panel: Temperature dependence of the unit-cell dimensions of the $\mathrm{La}_{0.9} \mathrm{Sr}_{0.1} \mathrm{MnO}_{3}$ single crystal. Lower panel: Temperature dependence of the dc resistivity (semi-logarithmic, left axis) and the inverse magnetic susceptibility, $1 / \chi$, in an external field of $H=1000 \mathrm{Oe}$ (right axis). The right inset shows the $O O^{\prime}$ transition of $\rho_{\mathrm{dc}}$ on linear scales, while the left inset displays the magnetic ac susceptibility $\chi_{\mathrm{ac}}$. The $O O^{\prime}, O^{\prime} O^{\prime \prime}$ phase boundaries and $T_{\mathrm{CA}}$ are indicated by vertical dotted lines in all frames.

electric dc resistivity, $\rho_{\mathrm{dc}}$ (left axis), as a function of temperature. The slope of the $1 / \chi$ curve above and below $T_{O O^{\prime}}$ is almost the same, indicating a similar paramagnetic moment in both phases. ${ }^{4}$ A distinct step shows up at the phase transition with a slightly reduced paramagnetic Curie temperature in the $O^{\prime}$ phase. Therefore, the distorted unit cell seems to weaken the magnetic interaction. The dc resistivity also shows a step at about $340 \mathrm{~K}$ (cf. inset of Fig. 5), pointing toward a more insulating character of the $O^{\prime}$ phase, which could be understood with the enhanced structural distortion in this phase.

Spectroscopy of the phonon modes can elucidate the role of the lattice for the different phase transitions. In Fig. 6 we plot the temperature dependence of the eigenfrequencies of the stretching and external phonon modes of $\mathrm{La}_{0.9} \mathrm{Sr}_{0.1} \mathrm{MnO}_{3}$. Most likely, they are identified as the above-mentioned cubic modes, but one has to keep in mind that the correct orthorhombic description ${ }^{18}$ reveals no pure external, bending, or stretching character of these modes. The two insets display the optical conductivity $\sigma^{\prime}$ of these two modes at selected temperatures. At low temperatures the phonon linewidth decreases, reflecting the enhanced phonon lifetime. For the mode frequencies unusual shifts exceeding the spectral resolution of $1 \mathrm{~cm}^{-1}$ are observed, which we relate to the different structural and magnetic phases in the following.

During the bending mode vibration the oxygen ions are displaced in such away from the $\mathrm{Mn}$ ion that the Mn-O bonding angle is changed. For the neighboring $\mathrm{Mn}$ sites this
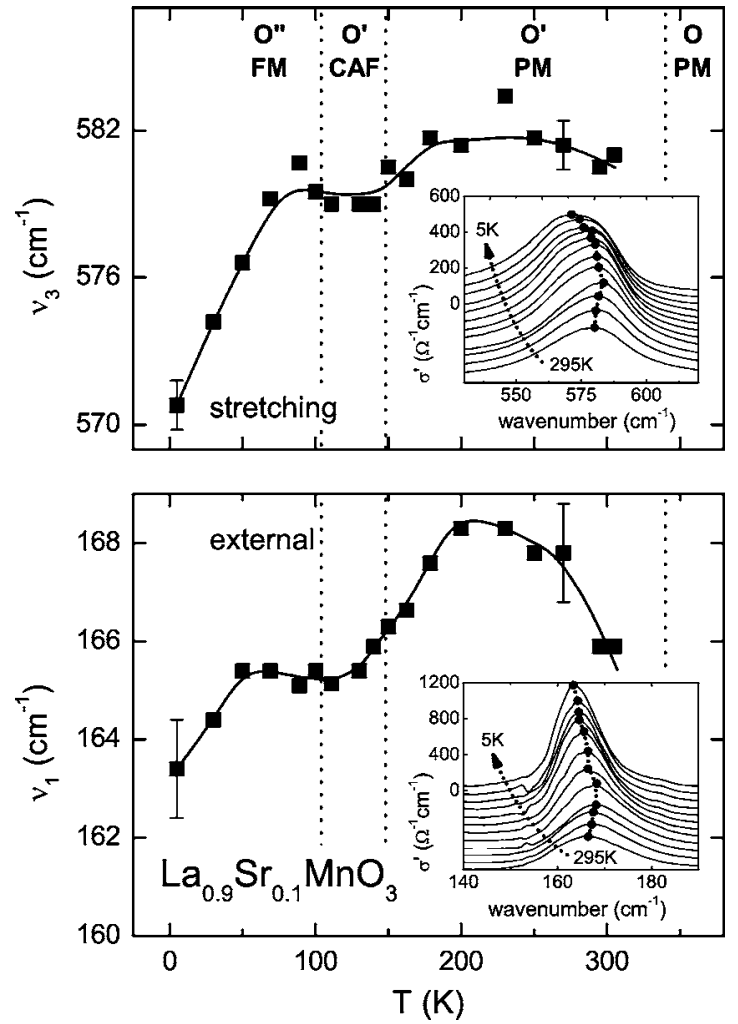

FIG. 6. Temperature dependence of the external and stretching phonon bands of $\mathrm{La}_{0.9} \mathrm{Sr}_{0.1} \mathrm{MnO}_{3}$ from room temperature to $5 \mathrm{~K}$. The phase boundaries $O / O^{\prime}, \mathrm{PM} / \mathrm{CAF}$, and $O^{\prime} / O^{\prime \prime}$ are indicated by the vertical dashed lines. The two insets show the optical conductivity spectra at selected temperatures between $295 \mathrm{~K}$ and $5 \mathrm{~K}$ for the two modes, respectively. For better clarity, $\sigma^{\prime}$ has been shifted for temperatures below $295 \mathrm{~K}$, each by the same amount.

means a constant distance, and no influence of the spin arrangement is expected. As can be seen from Fig. 3, the bending-type phonon group located around $350 \mathrm{~cm}^{-1}$ becomes increasingly broadened upon doping, and adjacent modes are difficult to separate. For this reason, we were not able to follow the doping dependence of the eigenmode frequency unambiguously.

The external mode (lower frame of Fig. 6) initially shows an increase upon lowering the temperature from $300 \mathrm{~K}$, which is the typical behavior expected for magnetic materials with an anharmonic lattice potential. ${ }^{43,44} \mathrm{~A}$ similar increase is observed for the stretching mode (upper frame of Fig. 6).

A remarkable reduction of $\nu(T)$ is visible for both, the external and the stretching modes, at the PM to CAF phase transition. For the latter, a coupling between spin and lattice seems to be quite natural, as for this vibration type, the Mn ions are displaced with respect to each other. A positive magnetic coupling between these ions seems to facilitate this motion. We may speculate that for the external mode the distance between neighboring $\mathrm{Mn}$ ions is also subjected to changes, and the magnetic exchange energy influences the force constant of this mode. The variation of the Mn-Mn distance may be either due to an out-of-phase motion of the neighboring $\mathrm{MnO}_{6}$ octahedra or to a mixing of stretching 
components to this commonly external labeled vibrational mode.

It is interesting to note that the decrease of $\nu(T)$ seems to start at about $230 \mathrm{~K}$, which is well above $T_{\mathrm{CA}}=148 \mathrm{~K}$. This indicates the existence of a new temperature scale above magnetic ordering, where magnetic effects already seem to play an important role for the lattice dynamics. Magnetic anomalies in this temperature range have very recently been reported for lightly doped LSMO and interpreted in terms of quenched disorder in the magnetic bond configuration, which leads to Griffiths-like effects. ${ }^{45}$ The magnetic disorder induced by the non-JT active $\mathrm{Mn}^{4+}$ ions $\left(\mathrm{Mn}^{3+}-\mathrm{Mn}^{4+}\right.$ doubleexchange bonds) is inevitably linked with structural disorder and may account for the observed features in the phononic behavior.

In the $O^{\prime \prime}$ phase below about $70 \mathrm{~K}$, the frequencies of the external and the stretching modes decrease strongly with decreasing temperature. On one hand, for this phase the JT distortion is reduced ${ }^{29}$ and the lattice resembles the structure of the $O$ phase. On the other hand, the canted spin arrangement is transformed into a parallel alignment, which enlarges the reduction of $\nu$ for the external and stretching modes in the presence of a strong magnetoelastic coupling. Indications of such a coupling between magnons and phonons have been found with neutron scattering for $x=0.09$ and $0.125 .^{8}$

For $x=0.11$ and 0.125 Takazawa et al. found variations in the bending mode phonon spectra at low temperatures, consistent with the orbitally ordered phase. In contrast to the present study they observed no anomalies for a sample with a Sr content $x=0.1$. As the phase diagram is quite sensitive to the hole concentration in this concentration regime, ${ }^{35}$ slight off-stoichiometries could account for this discrepancy.

\section{SUMMARY}

In conclusion, we show results of $\mathrm{x}$-ray and FIR measurements for $\mathrm{La}_{1-x} \mathrm{Sr}_{x} \mathrm{MnO}_{3}$ in the low-doping range up to $x$ $=0.125$. The temperature and doping dependence of the JT distortion are evaluated and related to the complex phase diagram. In particular, the temperature dependence for $x=0.1$ was analyzed in detail since the phase diagram for this concentration reveals a complex sequence of different phases. This enables us to study the interplay of the electronic, magnetic, and lattice degrees of freedom.

As $x$ increases, the $\mathrm{x}$-ray spectra exhibit that the strength of the JT distortion at RT is continuously reduced. For $x$ $\approx 0.11$ the lattice symmetry is changed from the distorted $O^{\prime}$ phase to the undistorted $O$ phase.

The various phonon modes prove to be sensitive to the local symmetry changes of the lattice. With increasing $\mathrm{Sr}$ doping, the distinct phonon structure of the parent $\mathrm{LaMnO}_{3}$ compound declines and merges into three groups of phonons. Especially the fine structured group of bending resonances of the Pnma symmetry disappears almost completely (cf. Fig. 3 ), in agreement with the above-mentioned weakening of the JT distortion, although this effect is facilitated by the increasing disorder. The doping dependence of the bending mode frequency can be understood within this JT scenario. A close inspection of the orthorhombic description of the stretching mode points to a predominant $b$-axis dependence of the JT effect in this particular case.

The $x=0.1$ sample is representative for the complex behavior of the low-doped part of the $\mathrm{La}_{1-x} \mathrm{Sr}_{x} \mathrm{MnO}_{3}$ phase diagram. From a comparison of the temperature dependence of the resistivity, magnetic susceptibility and the evaluated lattice parameters the different phases can be specified in detail. Upon lowering the temperature, the lattice and magnetic structure changes from $O / \mathrm{PM}$ to $O^{\prime} / \mathrm{PM}$, then to $O^{\prime} / \mathrm{CAF}$ and finally to $O^{\prime \prime} / \mathrm{FM}$. Moreover, the different structural and magnetic phase boundaries are observed in the temperature dependence of the phonon excitation energies. The irregular behavior of the eigenfrequencies of the external and stretching mode at the magnetic transition suggests a magnon-phonon coupling. Possible mechanisms underlying this coupling are proposed for both modes, based on the particular motion of the magnetic manganese ion.

\section{ACKNOWLEDGMENTS}

We thank T. Kopp for his careful proofreading of the manuscript. This work was partly supported by the BMBF under Contract No. 13N6917/Elektronische Korrelationen und Magnetismus, through SFB 484 (Augsburg) and by the European Community via INTAS 30850.
${ }^{1}$ M. Imada, A. Fujimori, and Y. Tokura, Rev. Mod. Phys. 70, 1039 (1998).

${ }^{2}$ E. Dagotto, T. Hotta, and A. Moreo, Phys. Rep. 344, 1 (2001).

${ }^{3}$ M. Paraskevopoulos, F. Mayr, J. Hemberger, A. Loidl, R. Heichele, D. Maurer, V. Müller, A. A. Mukhin, and A. M. Balbashov, J. Phys.: Condens. Matter 12, 3993 (2000).

${ }^{4}$ M. Paraskevopoulos, F. Mayr, Ch. Hartinger, A. Pimenov, J. Hemberger, P. Lunkenheimer, A. Loidl, A. A. Mukhin, V. Y. Ivanov, and A. M. Balbashov, J. Magn. Magn. Mater. 211, 118 (2000).

${ }^{5}$ J. Hemberger, A. Krimmel, T. Kurz, H.-A. Krug von Nidda, V. Y. Ivanov, A. A. Mukhin, A. M. Balbashov, and A. Loidl, Phys. Rev. B 66, 094410 (2002).
${ }^{6}$ J. Geck, P. Wochner, D. Bruns, B. Büchner, U. Gebhardt, S. Kiele, P. Reutler, and A. Revcolevschi, Phys. Rev. B 69, 104413 (2004).

${ }^{7}$ H. Nojiri, K. Kaneko, M. Motokawa, K. Hirota, Y. Endoh, and K. Takahashi, Phys. Rev. B 60, 4142 (1999).

${ }^{8}$ F. Moussa, M. Hennion, F. Wang, P. Kober, J. RodríguezCarvajal, P. Reutler, L. Pinsard, and A. Revcolevschi, Phys. Rev. B 67, 214430 (2003).

${ }^{9}$ R. Senis, V. Laukhin, B. Martínez, J. Fontcuberta, X. Obradors, A. A. Arsenov, and Y. M. Mukovskii, Phys. Rev. B 57, 14680 (1998).

${ }^{10}$ R. Klingeler, J. Geck, R. Gross, L. Pinsard-Gaudart, A. Revcolevschi, S. Uhlenbruck, and B. Büchner, Phys. Rev. B 65, 
174404 (2002)

${ }^{11}$ H. Kawano, R. Kajimoto, M. Kubota, and H. Yoshizawa, Phys. Rev. B 53, R14709 (1996).

${ }^{12}$ F. Mayr, Ch. Hartinger, M. Paraskevopoulos, A. Pimenov, J. Hemberger, A. Loidl, A. A. Mukhin, and A. M. Balbashov, Phys. Rev. B 62, 15673 (2000).

${ }^{13}$ Ch. Hartinger, F. Mayr, J. Deisenhofer, A. Loidl, and T. Kopp, Phys. Rev. B 69, 100403(R) (2004).

${ }^{14} \mathrm{Ch}$. Hartinger, F. Mayr, A. Loidl, and T. Kopp, cond-mat/ 0406123.

${ }^{15}$ Ch. Hartinger, F. Mayr, A. Loidl, and T. Kopp, 71, 184421 (2005).

${ }^{16}$ Ch. Hartinger, F. Mayr, A. Loidl, and T. Kopp, Phys. Rev. B 70, 134415 (2004).

${ }^{17}$ I. Fedorov, J. Lorenzana, P. Dore, G. De Marzi, P. Maselli, P. Calvani, S.-W. Cheong, S. Koval, and R. Migoni, Phys. Rev. B 60, 11875 (1999).

${ }^{18}$ I. S. Smirnova, Physica B 262, 247 (1999).

${ }^{19}$ G. V. Subba Rao, C. N. R. Rao, and J. R. Ferraro, Appl. Spectrosc. 24, 436 (1970).

${ }^{20}$ J. H. Jung, K. H. Kim, H. J. Lee, J. S. Ahn, N. J. Hur, T. W. Noh, M. S. Kim, and J.-G. Park, Phys. Rev. B 59, 3793 (1999).

${ }^{21}$ A. Paolone, P. Roy, A. Pimenov, A. Loidl, O. K. Mel'nikov, and A. Y. Shapiro, Phys. Rev. B 61, 11255 (2000).

${ }^{22}$ K. Tobe, T. Kimura, Y. Okimoto, and Y. Tokura, Phys. Rev. B 64, 184421 (2001).

${ }^{23}$ M. A. Quijada, J. R. Simpson, L. Vasiliu-Doloc, J. W. Lynn, H. D. Drew, Y. M. Mukovskii, and S. G. Karabashev, Phys. Rev. B 64, 224426 (2001).

${ }^{24}$ A. Takazawa, H. Nojiri, K. Nagasaka, K. Hirota, and Y. Endoh, J. Phys. Soc. Jpn. 70, 902 (2001).

${ }^{25}$ V. B. Podobedov, A. Weber, D. B. Romero, J. P. Rice, and H. D. Drew, Phys. Rev. B 58, 43 (1998).

${ }^{26}$ V. B. Podobedov, A. Weber, D. B. Romero, J. P. Rice, and H. D. Drew, Solid State Commun. 105, 589 (1998).

${ }^{27}$ E. Granado, N. O. Moreno, A. García, J. A. Sanjurjo, C. Rettori, I. Torriani, S. B. Oseroff, J. J. Neumeier, K. J. McClellan, S.-W. Cheong, and Y. Tokura, Phys. Rev. B 58, 11435 (1998).
${ }^{28}$ W. Reichardt and M. Braden, Physica B 263-264, 416 (1999).

${ }^{29}$ D. N. Argyriou, J. F. Mitchell, C. D. Potter, D. G. Hinks, J. D. Jorgensen, and S. D. Bader, Phys. Rev. Lett. 76, 3826 (1996).

${ }^{30}$ B. Dabrowski, X. Xiong, Z. Bukowski, R. Dybzinski, P. W. Klamut, J. E. Siewenie, O. Chmaissem, J. Shaffer, C. W. Kimball, J. D. Jorgensen, and S. Short, Phys. Rev. B 60, 7006 (1999).

${ }^{31}$ J. F. Mitchell, D. N. Argyriou, C. D. Potter, D. G. Hinks, J. D. Jorgensen, and S. D. Bader, Phys. Rev. B 54, 6172 (1996).

${ }^{32}$ D. Louca, T. Egami, E. L. Brosha, H. Röder, and A. R. Bishop, Phys. Rev. B 56, R8475 (1997).

${ }^{33}$ Q. Huang, A. Santoro, J. W. Lynn, R. W. Erwin, J. A. Borchers, J. L. Peng, and R. L. Greene, Phys. Rev. B 55, 14987 (1997).

${ }^{34}$ J. Rodríguez-Carvajal, M. Hennion, F. Moussa, A. H. Moudden, L. Pinsard, and A. Revcolevschi, Phys. Rev. B 57, R3189 (1998).

${ }^{35}$ D. E. Cox, T. Iglesias, E. Moshopoulou, K. Hirota, K. Takahashi, and Y. Endoh, Phys. Rev. B 64, 024431 (2001).

${ }^{36}$ T. Chatterji, B. Ouladdiaf, P. Mandal, B. Bandyopadhyay, and B. Ghosh, Phys. Rev. B 66, 054403 (2002).

${ }^{37}$ A. M. Balbashov, S. G. Karabashev, Y. M. Mukovskiy, and S. A. Zverkov, J. Cryst. Growth 167, 365 (1996).

${ }^{38}$ P. Mandal, B. Bandyopadhyay, and B. Ghosh, Phys. Rev. B 64, 180405(R) (2001).

${ }^{39}$ A. K. Bogush, V. I. Pavlov, and L. V. Balyko, Cryst. Res. Technol. 18, 589 (1983).

${ }^{40}$ M. Couzi and P. V. Huong, J. Chim. Phys. Phys.-Chim. Biol. 69, 1339 (1972).

${ }^{41}$ L. Pinsard, J. Rodríguez-Carvajal, and A. Revcolevschi, J. Alloys Compd. 262-263, 152 (1997).

${ }^{42}$ L. Pinsard, J. Rodríguez-Carvajal, A. H. Moudden, A. Anane, A. Revcolevschi, and C. Dupas, Physica B 234-236, 856 (1997).

${ }^{43}$ K. Wakamura, T. Arai, and K. Kudo, J. Phys. Soc. Jpn. 41, 130 (1976).

${ }^{44}$ K. Wakamura and T. Arai, J. Appl. Phys. 63, 5824 (1988).

${ }^{45}$ J. Deisenhofer, D. Braak, H.-A. K. von Nidda, J. Hemberger, R. Eremina, V. Ivanshin, A. Balbashov, A. Loidl, T. Kimura, and Y. Tokura, cond-mat/0501443. 